\title{
Safety and Efficacy of Tien-Hsien Liquid Practical in Patients with Refractory Metastatic Breast Cancer: A Randomized, Double-Blind, Placebo-Controlled, Parallel-Group, Phase IIa Trial
}

\author{
Wen-Hung Kuo, ${ }^{1}$ Chien-An Yao, ${ }^{2}$ Chih Hui Lin, ${ }^{3}$ and King-Jen Chang ${ }^{1,4}$ \\ ${ }^{1}$ Department of General Surgery, National Taiwan University Hospital, Taipi, Taiwan \\ ${ }^{2}$ Department of Family Medicine, National Taiwan University Hospital, Taipi, Taiwan \\ ${ }^{3}$ Gerent Biotech Co., Ltd., Taichung, Taiwan \\ ${ }^{4}$ Department of Surgery, Cheng Ching General Hospital, Taichung, Taiwan
}

Correspondence should be addressed to King-Jen Chang, kingjenchang@163.com

Received 15 December 2011; Accepted 18 January 2012

Academic Editor: Wolfgang Weidenhammer

Copyright (C) 2012 Wen-Hung Kuo et al. This is an open access article distributed under the Creative Commons Attribution License, which permits unrestricted use, distribution, and reproduction in any medium, provided the original work is properly cited.

To evaluate the safety and efficacy of Tien-Hsien Liquid Practical (THL-P), a Chinese herbal mixture, in patients with refractory metastatic breast cancer, we performed a randomized, double-blind, placebo-controlled, parallel-group, phase IIa pilot trial. Patients were randomly assigned to either receive THL-P or matching placebo and followed up every 4 weeks for 24 weeks. The primary endpoint was changes in the global health status/quality of life (GHS/QOL) scale. The secondary endpoints were changes in functional and symptom scales, immunomodulating effects, and adverse events. Sixty-three patients were enrolled between June 2009 and June 2011. The intent-to-treat population included 28 patients in the THL-P group and 11 patients in the placebo group. Compared to the placebo group, the THL-P group had significant improvement from baseline to last visit in GHS/QOL (41.7 versus -33.3; $P<0.05)$, CD3, CD4/CD8, CD19, CD16+56 positive cells $(P<0.05)$, and higher levels of physical, role, emotional, and cognitive functioning, as well as decreased fatigue and systemic side effects. Treatment-related adverse events were mild constipation and localized itching, and no serious adverse events were reported. THL-P appears to be a safe alternative adjuvant treatment for patients with refractory metastatic breast cancer, as it effectively improves QOL and palliates cancer-related symptoms.

\section{Introduction}

Breast cancer, a leading cause of morbidity and mortality among women, was estimated to account for $23 \%$ ( 1.38 million) of new cancer cases and $14 \%(458,400)$ of cancer deaths worldwide in 2008 [1, 2]. In Taiwan, breast cancer was the most common cancer among women, with an incidence of 9,049 cases per 100,000 population, and the fourth leading cause of cancer-related deaths in 2008 [3]. Despite advances in the treatment of breast cancer, it is estimated that 30-50\% of women initially diagnosed with earlier stages of breast cancer eventually develop metastatic disease [4]. Unfortunately, breast cancer has a poor prognosis following metastasis, and women with metastatic breast cancer have a limited survival of 18-24 months and a 5-year survival rate of approximately $20 \%[2,5]$. The progression to metastatic disease is further exacerbated by the increasing use of chemotherapy in earlystage breast cancer, which has led to a corresponding increase in the number of metastatic breast cancer cases refractory to conventional treatments [4].

The currently available conventional treatment options for metastatic breast cancer include cytotoxic chemotherapy, endocrine/hormonal therapy, targeted biological therapy, radiation therapy, bisphosphonates, or combinations of these, and surgery in some cases $[2,4,5]$. However, there is no global consensus and guidelines for the treatment of metastatic 
breast cancer, and treatment is highly individualized and varies worldwide. Furthermore, due to the poor prognosis of metastatic breast cancer, the main goals of treatment are palliative rather than curative. Specifically, treatment is aimed at prolonging progression-free survival (PFS) and overall survival (OS), managing or reducing disease symptoms, and achieving the best quality of life (QOL) $[4,5]$. However, the significant side effects, potential for the development of treatment resistance, and limited survival benefit associated with conventional treatments have prompted the use of complementary and alternative medicine (CAM) in patients with advanced malignancies [4, 6-8].

The use of CAM has been on the rise, especially among patients with life-threatening diseases, such as cancer [9]. In fact, it has been estimated that $48-98 \%$ of breast cancer patients use CAM, and the most commonly used CAM is herbal medicine [10-13]. There are numerous reasons cited by breast cancer patients for using CAM, including strengthening the immune system, increasing QOL, treating or preventing the recurrence of cancer, stabilizing current condition, alleviating cancer-related symptoms, assisting conventional treatments, relieving symptoms and stress associated with side effects of conventional treatments, providing a feeling of control over life, reducing stress and detoxification, and compensating for failed conventional medical treatments [10, 12, 13]. Although CAM is widely used by breast cancer patients, their safety and efficacy have been studied in very few randomized and controlled clinical trials [13].

Tien-Hsien Liquid Practical (THL-P), a Chinese herbal mixture, has been used as a CAM for over ten years. Recently, THL-P has been shown to have strong immunomodulating and anticancer effects via a number of preclinical in vitro and in vivo experiments $[6,7,9,14,15]$. Specifically, THL-P has been shown to modulate the antigen-stimulated proliferation response and cytokine production of T-lymphocytes, inhibit cell growth and induce apoptosis in various human cancer cell lines and possess antitumour, antiangiogenic, antimetastatic effects $[6,7,9,14,15]$. Based on these promising findings, we performed a randomized, double-blind, placebo-controlled, parallel-group, phase IIa pilot trial to evaluate the safety and efficacy profiles of THL-P in patients with metastatic breast cancer refractory to conventional treatments.

\section{Methods}

2.1. Study Design. This study was a randomized, doubleblind, placebo-controlled, parallel-group, phase IIa pilot trial in patients with metastatic breast cancer refractory to conventional treatment modalities. The primary outcome was the changes from baseline to posttreatment evaluations in the global health status/quality of life (GHS/QOL) standardized scale, assessed by the self-administered European Organization for Research and Treatment of Cancer Quality of Life Questionnaire-Core 30 (EORTC-QLQ-C30). The secondary outcomes included changes in functional and symptom scales, as well as the single items, of the EORTC-QLC-Q30 and -Breast Cancer 23 (BR23), immunomodulating effects of treatment on lymphocytes, and treatment-related adverse events. The study was approved by the Institutional Review Board (IRB) of the National Taiwan University Hospital, and written informed consents were obtained from all patients prior to entering the trial.

2.2. Participants. Subjects were recruited at the National Taiwan University Hospital in Taipei, Taiwan between June 2009 and June 2011. The inclusion criteria were women, aged 20 to 80 years old, who had histologically or cytologically confirmed breast cancer with clinical evidence of progressive metastatic disease and any one of the following: (a) no satisfactory response after primary or salvage treatment (i.e., chemotherapy, radiotherapy, surgery, or other approved therapies, such as target therapy or immunotherapy) and (b) no intention of accepting additional conventional treatments. Subjects were included provided that they had adequate bone marrow function with an absolute neutrophil count $\geq 1000 / \mu \mathrm{L}$, hemoglobin count $\geq 8 \mathrm{~g} / \mathrm{dL}$, and platelet count $>75,000 / \mu \mathrm{L}$, and liver and renal function with total serum bilirubin $<3 \mathrm{mg} / \mathrm{dL}$ and serum creatinine $<2 \mathrm{mg} / \mathrm{dL}$, respectively. Subjects of childbearing potential had to agree to use medically accepted means of contraception during the participation of the study. Subjects must also have an estimated life expectancy of at least 4 weeks. All subjects had to give written informed consent to participate in the study.

Subjects were excluded if they have ever received radiotherapy, endocrine therapy, antineoplastic drugs, or hormonal agents as adjuvant treatment or therapy for metastatic breast cancer within 2 weeks prior to entering the study. Additional exclusion criteria included uncontrolled infections, history of autoimmune disease (i.e., lupus erythematosus, ankylosing spondylosis, scleroderma or multiple sclerosis), prior history of other malignancies, with the exception of skin basal cell carcinoma, within 3 years of study entry, or any other serious diseases or medical history considered by the investigator to place the subject at increased risk. Subjects with aspartate aminotransferase (AST) and alanine aminotransferase (ALT) levels above five times the upper limit of normal values, or if liver metastases were present, above ten times the upper limit of normal values, were excluded. Women who were lactating, pregnant, or planning to become pregnant were also excluded. Lastly, subjects were not eligible if they had participated in any other investigational study within 4 weeks of study entry.

Subjects were withdrawn from the study if any of the following criteria were met: (a) subject decided to withdraw her informed consent, (b) the investigator considered the subject to be no longer physically or psychologically capable of remaining in the study, (c) subject refused to proceed with critical measures for the study endpoints, defined as all variables required for the primary endpoint analysis, or (d) subject developed adverse effects that the investigator considered as warranting discontinuation of the study treatment.

2.3. Treatment Groups. The study group received 1 vial $(20 \mathrm{~mL})$ of THL-P oral solution 3 times per day for 24 weeks. THL-P is an aqueous preparation that consists of extracts from 14 Chinese medicinal herbs, 11 of which are active 
ingredients and the remaining three are flavoring ingredients. The active ingredients of THL-P oral solution (Sheng Foong Co., Ltd., I-Lan County, Taiwan) are Atractylodes macrocephala (250 mg/mL), Astragalus membranaceus (330 mg/ $\mathrm{mL})$, Taraxacum mongolicum $(500 \mathrm{mg} / \mathrm{mL})$, Poria cocos (330 mg/mL), Ligusticum chuanxiong $(250 \mathrm{mg} / \mathrm{mL})$, Ligustrum lucidum $(250 \mathrm{mg} / \mathrm{mL})$, Codonopsis pilosula $(250 \mathrm{mg} /$ $\mathrm{mL})$, Glycyrrhiza uralensis $(160 \mathrm{mg} / \mathrm{mL})$, Hedyotis diffusa $(330 \mathrm{mg} / \mathrm{mL})$, Pseudostellaria heterophylla $(250 \mathrm{mg} / \mathrm{mL})$, and Viola philippica $(160 \mathrm{mg} / \mathrm{mL})$. The compositions, as well as the pharmacological and immunological effects, of these ingredients, have been previously described $[14,15]$. The control group received 1 vial $(20 \mathrm{~mL})$ of a matched placebo 3 times per day for 24 weeks. The matched placebo consisted of food-grade flavoring ingredients, which ensured a similar appearance, taste, and odor to the THL-P oral solution, and was dispensed in a similar opaque plastic bottle.

The investigators attempted to minimize the use of concomitant treatments in each subject throughout the study. If concomitant treatments were deemed necessary by the investigator, then it was ensured that a stable dose and therapy type were maintained throughout the study to minimize potential interference with the study endpoint assessments. Antipyretics on the day of injection were permitted, whereas all cancer treatments, with the exception of non-studyrelated local lesions palliative radiation therapy, were prohibited during the study.

2.4. Randomization and Blinding. Treatment allocation was performed prior to site initiation, and each patient was assigned a unique number based on the order of enrolment. Patients meeting the eligibility criteria were randomly assigned in a 2:1 ratio to either the THL-P treatment or placebo group. Randomization was achieved with the use of a permuted-block randomization algorithm with a block size of 6 in SAS 9.0 software (SAS Institute Inc., Cary, NC, USA), where a list of sequential numbers was generated with each number randomly assigned to a group. All patients, caregivers, investigators, and outcome assessors were blinded to treatment assignment.

2.5. Measurement of Outcomes. After randomization, several examinations were conducted at baseline and followed up every 4 weeks for 24 weeks. The examinations included selfadministered EORTC-QLQ-C30, EORTC-QLC-Q30 and -Breast Cancer 23 (BR23), body weight, collection of blood for assessment of T-lymphocyte activity, biochemistry, and hematology tests.

The primary endpoint was to evaluate the efficacy of the treatment in improving the QOL of patients from baseline to last visit. The changes in the GHS/QOL scale were assessed by the self-administered EORTC-QLQ-C30. The secondary outcomes evaluated the efficacy and safety of the treatment. The efficacy of the treatment was evaluated between baseline and last visit via the following measures: (a) changes and maximum improvements in the functional and symptom standardized scales, as well as the single items, of the EORTCQLC-Q30 and -Breast Cancer 23 (BR23), (b) changes in body weight, (c) immunomodulating effects of treatment on lymphocytes, as determined via phenotypic analyses of lymphocytes by flow cytometry (FACSCalibur, BD) with monoclonal antibodies for CD3, CD4, CD8, CD19, and CD16+56, and BD MultiSET software. The safety of the treatment was determined from the reports of adverse events.

2.6. Statistical Analyses. The sample size for this study was arbitrarily determined to be 60 subjects for this pilot study to collect useful information for further study in the future. Demographic and clinical characteristics at baseline were analyzed according to randomized treatment groups. Primary and secondary endpoints were analyzed based on the intent-to-treat (ITT) population, which was defined as all randomized patients that received any treatment. Data of continuous variables are presented as mean \pm standard deviation. When the normality of these variables cannot be assumed, the data are presented as median (interquartile range). Data of categorical variables are presented as numbers (percentages). Statistical comparisons between continuous variables were made using the independent, two-sample $t$-test. The Mann-Whitney $U$ test was used to compare independent groups of data that were not normally distributed. A Fisher's exact test was used for comparisons of categorical variables. A parametric Student's paired $t$-test or nonparametric Wilcoxon signed ranks test were used to compare differences before and after treatment in each group. For all analyses, a two-sided $P$ value of $<0.05$ was considered significant. Our primary hypothesis is that there would be a significant improvement in the QOL between baseline and lastvisit assessments in the THL-P versus placebo groups. Statistical analyses were performed using SPSS 15.0 statistics software (SPSS Inc, Chicago, IL, USA) and SAS 9.0 (SAS Institute Inc., Cary, NC, USA).

\section{Results}

3.1. Study Population. A total of 63 patients entered our trial between June 2009 and June 2011, and 19 patients were excluded due to lack of confidence in the treatment $(n=10)$, hospital transfer $(n=2)$, and treatment refusal $(n=7)$. Overall, the screening failure rate was $30.2 \%$, and 44 patients were randomly assigned in a 2:1 ratio to either the THL-P group $(n=30)$ or the placebo group $(n=14)$ (Figure 1$)$. Of these 44 patients, 39 had metastasis in bone, 19 in liver, 18 in lung, and 4 in brain. The demographic and clinical characteristics of the 44 randomized patients are summarized in Table 1 . There were no statistically significant differences between the THL-P and placebo groups in age, height, body weight, stage of cancer (i.e., according to the TNM classification), duration of onset, and the scales and single items of the EORTO-QLQ-C30. Of the patients that were randomized to treatment, 31 patients $(70.5 \%)$ did not complete the study. Their withdrawal reasons were listed in Figure 1. In the THL-P group, 13 patients completed the study, while none of the patients in the placebo group completed the study. Interestingly, all of the participants in the placebo group withdrew prior to week 12, whereas only 11 


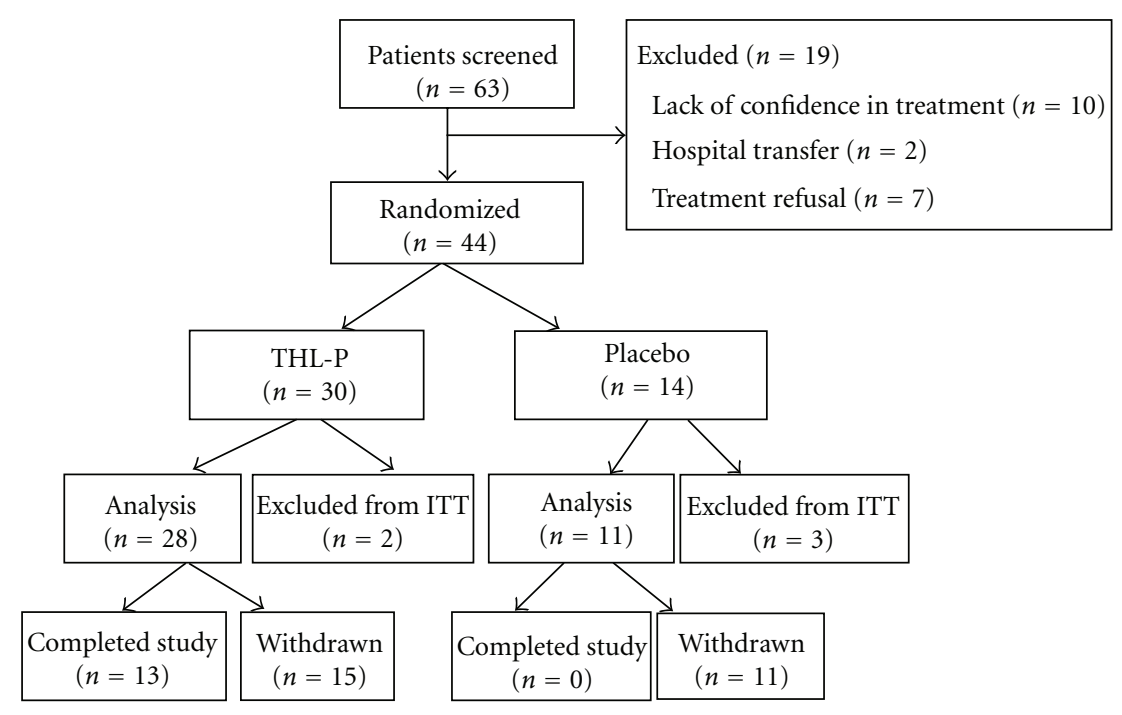

FIGURE 1: Flow diagram of the randomized, double-blind, placebo-controlled, parallel-group phase IIa clinical trial comparing Tien Hsien liquid practical (THL-P) to placebo for safety and efficacy. A total of 63 patients entered our trial between June 2009 and June 2011 . Nineteen patients were excluded due to lack of confidence in the treatment $(n=10)$, hospital transfer $(n=2)$, and treatment refusal $(n=7)$. Fortyfour patients were randomly assigned in a $2: 1$ ratio to either the THL-P group $(n=30)$ or the placebo group $(n=14)$. Thirteen patients in the THL-P group and none in the placebo group had completed the study.

participants (39.3\%) in the THL-P group withdrew prior to week 12. The ITT population was comprised of 39 patients, with 28 patients in the THL-P group and 11 patients in the placebo group (Figure 1).

3.2. Efficacy. The primary endpoint, in particular, changes in the GHS/QOL standardized scale between the baseline and last visit of the ITT population, is presented in Figure 2. There was a significant difference between the THL-P and placebo group with respect to the change in the GHS/QOL scale from the baseline and last visit (41.69 versus -33.33; $P<0.001$, Table 2, Figure 2). These findings indicate that patients administered THL-P had a higher and improved QOL than those receiving a matched placebo. Thus, THL-P appears to be efficacious in improving the QOL of patients with refractory metastatic cancer.

Table 2 presents the primary endpoints for changes in the standardized functional and symptom scales, as well as the single items, of the EORTO-QLQ-C30. There were significant differences in the changes from baseline to last visit in four out of five functional scales (i.e., physical, role, emotional, and cognitive functions), as well as one symptom scale (i.e., fatigue), between the THL-P and placebo groups $(P<$ $0.05)$. Indeed, the THL-P group appears to have improvements in these scales compared to the placebo group. There were no significant differences from baseline to last visit in the single items (i.e., dyspnoea, insomnia, loss of appetite, constipation, diarrhoea, and financial difficulties) of the EORTO-QLQ-C30 between the groups.

The secondary endpoints for changes in the standardized functional and symptom scales the EORTO-QLQ-BR23 are presented in Table 3. There was a significant decrease in the changes from baseline to last visit in the "systemic therapy

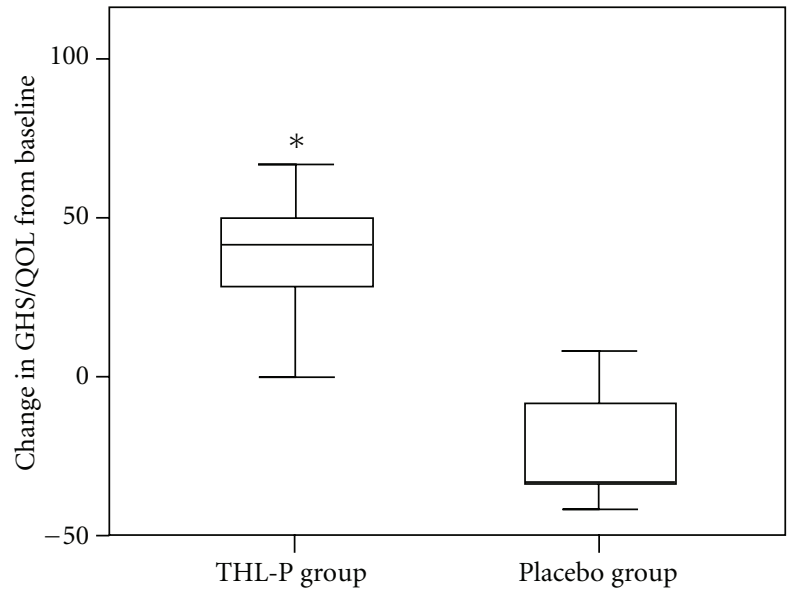

FIGURE 2: Primary endpoint of the intent-to-treat population. Changes in the standardized score of the global health/quality of life (GHS/QOL) scale of the European Organization for Research and Treatment of Cancer Quality of Life Questionnaire-Core 30 (EORTO-QLQ-C30) in the intent-to-treat population. Treatment of Cancer Quality of Life Questionnaire-Core 30 (EORTO-QLQC30). Values represent medians (interquartile ranges). ${ }^{*} P<0.05$ versus placebo (Mann-Whitney $U$ test).

side effects" scale between the THL-P and placebo groups $(P<0.05)$. Lastly, there were no significant differences in the body weights (i.e., \% change) of the two groups between the baseline and last visit (Table 3).

3.3. Safety. The treatment-related adverse events reported during the study were mild constipation $(n=6)$ and localized itching $(n=1)$. Constipation was relieved by increasing 
TABLE 1: Demographic and clinical characteristics of patients with refractory metastatic breast cancer at baseline.

\begin{tabular}{|c|c|c|c|}
\hline & THL-P $(n=30)$ & Placebo $(n=14)$ & $P$ value \\
\hline \multicolumn{4}{|l|}{ Demographics } \\
\hline Age (years) ${ }^{\mathrm{a}}$ & $60.7 \pm 9.5$ & $58.7 \pm 7.6$ & 0.507 \\
\hline Height $(\mathrm{cm})^{\mathrm{a}}$ & $155.3 \pm 5.9$ & $156.5 \pm 5.4$ & 0.512 \\
\hline Weight $(\mathrm{kg})^{\mathrm{a}}$ & $57.6 \pm 8.1$ & $59.3 \pm 10.5$ & 0.554 \\
\hline \multicolumn{4}{|l|}{ T stage, $n(\%)^{\mathrm{b}}$} \\
\hline $\mathrm{T} 1$ & $4(13.4)$ & $1(7.2)$ & \multirow{4}{*}{0.968} \\
\hline $\mathrm{T} 2$ & $12(40.0)$ & $7(50.0)$ & \\
\hline T3 & $7(23.3)$ & $3(21.4)$ & \\
\hline $\mathrm{T} 4$ & $7(23.3)$ & $3(21.4)$ & \\
\hline \multicolumn{4}{|l|}{ Lymph nodes, $n(\%)^{\mathrm{b}}$} \\
\hline No & $10(33.3)$ & $3(12.4)$ & \multirow{4}{*}{0.306} \\
\hline N1 & $6(20.0)$ & $2(14.3)$ & \\
\hline $\mathrm{N} 2$ & $4(13.4)$ & $2(14.3)$ & \\
\hline N3 & $10(33.3)$ & $7(50.0)$ & \\
\hline \multicolumn{4}{|l|}{ Metastasis, $n(\%)^{\mathrm{b}}$} \\
\hline M1 & $30(100)$ & $14(100)$ & N/A \\
\hline Duration of onset (years) ${ }^{c}$ & $3.5(2.0,7.0)$ & $4.1(2.7,5.4)$ & 0.970 \\
\hline \multicolumn{4}{|l|}{ EORTO-QLQ-C30 } \\
\hline GHS/QOL & $37.5(16.7,50.0)$ & $50.0(25.0,66.7)$ & 0.082 \\
\hline \multicolumn{4}{|l|}{ Functional scales } \\
\hline Physical $^{c}$ & $60.0(46.7,73.3)$ & $70.0(46.7,86.7)$ & 0.336 \\
\hline Role $^{c}$ & $66.7(33.3,83.3)$ & $66.7(33.3,100.0)$ & 0.643 \\
\hline Emotional $^{\mathrm{c}}$ & $66.7(50.0,83.3)$ & $75.0(58.3,91.7)$ & 0.469 \\
\hline Cognitive $^{\mathrm{c}}$ & $66.7(50.0,83.3)$ & $83.3(66.7,83.3)$ & 0.118 \\
\hline Social $^{c}$ & $66.7(33.3,100.0)$ & $66.7(66.7,100.0)$ & 0.711 \\
\hline \multicolumn{4}{|l|}{ Symptom scales } \\
\hline Fatigue $^{c}$ & $55.6(33.3,66.7)$ & $38.9(22.2,55.6)$ & 0.273 \\
\hline Nausea and vomiting ${ }^{c}$ & $0(0,16.7)$ & $0(0,0)$ & 0.362 \\
\hline Pain $^{c}$ & $33.3(0,66.7)$ & $8.3(0,50.0)$ & 0.382 \\
\hline \multicolumn{4}{|l|}{ Single items } \\
\hline Dyspnoea $^{c}$ & $33.3(0,66.7)$ & $0(0,33.3)$ & 0.069 \\
\hline Insomnia $^{c}$ & $33.3(0,33.3)$ & $33.3(0,66.7)$ & 0.608 \\
\hline Appetite loss ${ }^{\mathfrak{c}}$ & $33.3(0,66.7)$ & $0(0,33.3)$ & 0.367 \\
\hline Constipation $^{c}$ & $0(0,33.3)$ & $0(0,33.3)$ & 0.403 \\
\hline Diarrhoea $^{c}$ & $0(0,0)$ & $0(0,33.3)$ & 0.523 \\
\hline Financial difficulties $^{c}$ & $33.3(0,33.3)$ & $33.3(0,33.3)$ & 0.746 \\
\hline
\end{tabular}

THL-P: Tien-Hsien liquid practical: EORTO-QLQ-C30: European Organization for Research and Treatment of Cancer Quality of Life Questionnaire-Core 30; GHS: global health status; QOL: quality of life.

$P$ values were determined via the independent, two sample $t$-test ${ }^{\mathrm{a}}$, Fisher's exact test ${ }^{\mathrm{b}}$, and Mann-Whitney $U$ test ${ }^{\mathrm{c}}$. Values are presented as mean \pm standard deviation $^{\mathrm{a}}$, number (percentage) $)^{\mathrm{b}}$, and median (interquartile) ${ }^{\mathrm{c}}$.

water intake, and itching subsided immediately after cessation of all medication. No nausea, vomiting, hair loss, diarrhoea, and heavy constipation were observed. Nine severe adverse events were reported by five participants. However, all serious adverse events were considered unrelated to THLP treatment by the IRB.

3.4. Immunomodulating Effects. There were significant differences in the changes from baseline to the last visit in $\mathrm{CD} 3$, CD4/CD8, CD19, and CD16+56 positive cells between the THL-P and placebo groups $(P<0.05)$ (Table 3$)$. Specifically, compared with placebo, THL-P appears to have elevated the levels of CD3, CD4/CD8, CD19, and CD16+56 positive cells.

\section{Discussion}

In patients with refractory metastatic breast cancer, the oral administration of $20 \mathrm{~mL}$ THL-P three times a day for 24 weeks significantly improved the QOL, increased the physical, role, emotional, and cognitive functioning, decreased fatigue and systemic therapy side effects, and had immunomodulating effects on lymphocytes. Furthermore, THL-P 
TABLE 2: Primary endpoints: Changes in the functional and symptom scales of EORTO-QLQ-C30 in the intent-to-treat population.

\begin{tabular}{|c|c|c|c|}
\hline & THL-P $(n=28)$ & Placebo $(n=11)$ & $P$-value \\
\hline \multicolumn{4}{|l|}{ Change from baseline } \\
\hline \multicolumn{4}{|l|}{ EORTO-QLQ-C30 } \\
\hline GHS/QOL & $41.7(29.2,50.0)^{\dagger}$ & $-33.3(-33.3,0)^{\dagger}$ & $<0.001^{*}$ \\
\hline \multicolumn{4}{|l|}{ Functional scales } \\
\hline Physical & $13.3(3.3,26.7)^{\dagger}$ & $0(-13.3,13.3)$ & $0.014^{*}$ \\
\hline Role & $0(0,41.7)^{\dagger}$ & $0(-16.7,0)$ & $0.018^{*}$ \\
\hline Emotional & $8.3(0,25.0)^{\dagger}$ & $0(-33.3,8.3)$ & $0.024^{*}$ \\
\hline Cognitive & $16.7(0,16.7)^{\dagger}$ & $0(-33.3,0)$ & $<0.001^{*}$ \\
\hline Social & $0(0,33.3)^{\dagger}$ & $0(-33.3,33.3)$ & 0.379 \\
\hline \multicolumn{4}{|l|}{ Symptom scales } \\
\hline Fatigue & $-22.2(-33.3,-11.1)^{\dagger}$ & $0(-11.1,22.2)$ & $<0.005^{*}$ \\
\hline Nausea and vomiting & $0(0,0)$ & $0(0,16.7)$ & 0.656 \\
\hline Pain & $0(-25.0,16.7)$ & $0(0,16.7)$ & 0.124 \\
\hline \multicolumn{4}{|l|}{ Single items } \\
\hline Dyspnoea & $0(-33.3,0)$ & $0(0,0)$ & 0.528 \\
\hline Insomnia & $0(0,16.7)$ & $0(0,33.3)$ & 0.569 \\
\hline Appetite loss & $0(0,0)$ & $0(0,0)$ & 0.633 \\
\hline Constipation & $0(-16.7,0)$ & $0(0,0)$ & 0.770 \\
\hline Diarrhoea & $0(0,33.3)$ & $0(0,33.3)$ & 0.866 \\
\hline Financial difficulties & $0(0,0)$ & $0(0,0)$ & 0.747 \\
\hline
\end{tabular}

THL-P: Tien-Hsien liquid practical; EORTO-QLQ-C30: European Organization for Research and Treatment of Cancer Quality of Life Questionnaire-Core 30. ${ }^{*} P<0.05$ versus placebo (Mann-Whitney $U$ test). ${ }^{\dagger} P<0.05$ versus baseline (Wilcoxon signed ranks test). Values are presented as median (interquartile).

TABLE 3: Secondary endpoints: changes in the functional and symptom scales of EORTO-QLQ-BR23, lymphocytes, and body weight in the intent-to-treat population.

\begin{tabular}{|c|c|c|c|}
\hline & THL-P $(n=28)$ & Placebo $(n=11)$ & $P$ value \\
\hline \multicolumn{4}{|l|}{ Change from baseline } \\
\hline \multicolumn{4}{|l|}{ EORTO-QLQ-BR23 } \\
\hline \multicolumn{4}{|l|}{ Functional scales ${ }^{\mathrm{a}}$} \\
\hline Body image & $0(0,33.3)^{\dagger}$ & $0(0,8.3)$ & 0.346 \\
\hline Sexual function & $0(0,0)$ & $0(0,0)$ & 0.591 \\
\hline Sexual enjoyment & $0(0,0)$ & $16.7(0,33.3)$ & 0.582 \\
\hline Future perspective & $16.7(0,33.3)^{\dagger}$ & $0(0,0)$ & 0.102 \\
\hline \multicolumn{4}{|l|}{ Symptom scales ${ }^{\mathrm{a}}$} \\
\hline Systemic therapy side effects & $-4.8(-23.8,0)^{\dagger}$ & $4.8(-4.8,9.5)$ & $0.010^{*}$ \\
\hline Breast symptoms & $0(-16.7,8.3)$ & $0(-8.3,0)$ & 0.450 \\
\hline Arm symptoms & $-5.6(-33.3,0)^{\dagger}$ & $0(-11.1,22.2)$ & 0.346 \\
\hline Upset by hair loss & $-33.3(-33.3,0)^{\dagger}$ & $0(-16.7,0)$ & 0.316 \\
\hline \multicolumn{4}{|l|}{ Lymphocytes (\%) } \\
\hline $\mathrm{CD} 3$ & $6.0(1.0,9.5)^{\dagger}$ & $-2.5(-6.0,-1.0)$ & $0.001^{*}$ \\
\hline $\mathrm{CD} 4$ & $0.0(-2.0,8.0)$ & $-1.5(-4.0,1)$ & 0.157 \\
\hline $\mathrm{CD} 8$ & $-1.5(-3.0,1.5)$ & $0.5(-2.0,2.0)$ & 0.387 \\
\hline $\mathrm{CD} 4 / \mathrm{CD} 8$ & $0.2(-0.1,0.5)^{\dagger}$ & $-0.1(-0.2,0.0)$ & $0.043^{*}$ \\
\hline CD19 & $3.0(0.0,7.5)^{\dagger}$ & $-2.0(-3.0,3.0)$ & $0.021^{*}$ \\
\hline $\mathrm{CD} 16+56$ & $8.0(3.0,11.0)^{\dagger}$ & $-4.0(-6.0,-3.0)^{\dagger}$ & $<0.001^{*}$ \\
\hline Body weight $(\%)^{\mathrm{b}}$ & $0.2 \pm 3.4$ & $0.3 \pm 4.0$ & 0.964 \\
\hline
\end{tabular}

THL-P: Tien Hsien liquid practical; EORTO-QLQ-BR23: European Organization for Research and Treatment of Breast Cancer-Specific Quality of Life Questionnaire.

${ }^{*} P<0.05$ versus placebo (Mann-Whitney $U$ test $^{\mathrm{a}}$ or independent, two-sample test $\mathrm{t}^{\mathrm{b}}$ ).

${ }^{\dagger} P<0.05$ versus baseline (Wilcoxon signed ranks test ${ }^{\mathrm{a}}$ or paired Student's $t$-test ${ }^{\mathrm{b}}$ ). Values are presented as median (interquartile) $)^{\mathrm{a}}$ and mean \pm standard deviation $^{\mathrm{b}}$. 
treatment did not induce any severe adverse events, and the only side effects reported were mild constipation and localized itching. Together, these findings suggest that THL-P is a safe and effective CAM for patients with refractory metastatic breast cancer.

Currently, no global consensus or guidelines exist for the treatment of patients with metastatic breast cancer, especially for those refractory to conventional treatments $[4,5]$. Furthermore, it is estimated that anywhere from 48 to $98 \%$ of all breast cancer patients use some form of CAM [10-13]. Interestingly, achieving the best quality of life (QOL) is one of the main goals of conventional treatments, as well as reasons for using CAM $[4,5,10,12,13]$. It was recently reported that the GHS/QOL scale, assessed via the EORTC-QLQ-C30, can serve as an important predictor of response to treatment, PFS, and OS in women with metastatic breast cancer [16]. In the present study, which used the same questionnaire to assess the GHS/QOL, as well as the function and symptom scales, found that THL-P was effective in improving the QOL and functions of refractory metastatic breast cancer patients. Given these positive findings, larger trials examining the effects of THL-P on other pertinent outcomes, such as PFS and OS, are warranted.

The use of CAM has been on the rise worldwide, especially among patients with life-threatening diseases, such as cancer [9]. There are also marked cultural differences in the way CAM is integrated into breast cancer treatment regiments [13]. In Asian populations, including Taiwan, the use of traditional Chinese medicines is very common and enjoys widespread popularity $[13,17]$. However, the use of CAM may be associated with severe adverse effects or CAM-drug interactions with conventional treatments for breast cancer, and consequently their safety and efficacy warrant investigation via randomized and controlled clinical trials $[13,18]$. In this randomized, double-blind, placebo-controlled, parallelgroup, phase IIa trial, it was found that THL-P was not only an effective CAM in patients with refractory metastatic breast cancer, but also a safe alternative adjuvant, since there were no serious adverse events reported with its use. Although these findings suggest that the use of THL-P is safe and effective, studies that examine the safety of using THL-P, as well as other CAMs, in combination with conventional treatments are needed.

In the present study, there were more withdrawal cases in the placebo group than in the THL-P treatment group. Given that this was a double-blinded study, where the patients, caregivers, investigators, and outcome assessors were blinded to treatment, we speculate that the significant difference in the number of withdrawal cases between the control and study groups suggests that THL-P may have possible therapeutic effects in patients with refractory metastatic breast cancer, which requires further investigation.

Although not directly studied, the therapeutic efficacy of THL-P in patients with metastatic breast cancer can be extended to its antimetastatic, antiangiogenic, and antitumour effects, as previously demonstrated via in vitro and in vivo preclinical studies [6]. Furthermore, corroborating the observations of the present study, THL-P was also found not to have any adverse effects on the body weights of immunocompromised mice [6]. In addition to its anticancer effects, THL-P was found to have immunomodulating effects, specifically in reducing cytokine production of $\mathrm{T}$ lymphocytes isolated from patients with recurrent aphthous ulcerations $[14,15]$. Herein, we also demonstrated that THL$\mathrm{P}$ has immunomodulating effects in patients with refractory metastatic breast cancer. Specifically, we found that there were marked differences in the changes from baseline to the last visit in CD3, CD4/CD8, CD19, and CD16+56 positive cells between the THL-P and placebo groups. Although our study provided the first clinical evidence for the safety and efficacy of using THL-P in refractory metastatic breast cancer patients, future studies assessing the specific mechanisms responsible for its immunomodulating effects in this population are warranted.

CAMs are widely used by breast cancer patients; however their safety and efficacy have been studied in very few randomized and controlled clinical trials $[8,13,19]$. The present study is one of a few to provide safety and efficacy evidence for a CAM that was beyond empirical evidence, case studies, and hypothetical physiological effects. Despite these important findings, there are several limitations in the present study, including that this was a single-site study with a small sample size and that THL-P is a compound with multiple ingredients. Therefore, further investigations on the exact therapeutic mechanisms for each ingredient of THL-P, as well as multiple site-, large-scale studies that confirm and extend the safety and efficacy findings of the present study, are warranted. However, it should be mentioned that, to our best knowledge, there are no studies reporting that the active ingredients of THL-P have any estrogenic effects or pose a risk for increased tumour growth [20-30].

\section{Conclusions}

Our findings suggest that an oral administration of $20 \mathrm{~mL}$ THL-P three times a day for 24 weeks significantly improves the QOL, increases the physical, role, emotional, and cognitive functioning, decreases fatigue and systemic therapy side effects, and has immunomodulating effects on lymphocytes in patients with refractory metastatic breast cancer. Additionally, THL-P treatment did not induce any severe adverse events. Together, these findings suggest that THL-P is a safe and effective CAM for patients with refractory metastatic breast cancer.

\section{Conflict of Interests}

The authors declare no conflict of interests.

\section{References}

[1] A. Jemal, F. Bray, M. M. Center, J. Ferlay, E. Ward, and D. Forman, "Global cancer statistics," CA Cancer Journal for Clinicians, vol. 61, no. 2, pp. 69-90, 2011.

[2] F. Cardoso and M. Castiglione, "Locally recurrent or metastatic breast cancer: ESMO clinical recommendations for diagnosis, treatment and follow-up," Annals of Oncology, vol. 20, supplement 4, pp. iv15-iv18, 2009. 
[3] Taiwan Cancer Registry, http://tcr.cph.ntu.edu.tw/uploadimages/Top\%2010\%20cancer\%20in\%20Taiwan\%202008.pdf.

[4] H. Roché and L. T. Vahdat, "Treatment of metastatic breast cancer: second line and beyond," Annals of Oncology, vol. 22, no. 5, pp. 1000-1010, 2011.

[5] Y. Fernández, J. Cueva, A. G. Palomo et al., "Novel therapeutic approaches to the treatment of metastatic breast cancer," Cancer Treatment Reviews, vol. 36, no. 1, pp. 33-42, 2010.

[6] J. S. Chia, J. L. Du, W. B. Hsu, A. Sun, C. B. Chiang, and W. B. Wang, "Inhibition of metastasis, angiogenesis, and tumor growth by chinese herbal cocktail Tien-Hsien Liquid," BMC Cancer, vol. 10, p. 175, 2010.

[7] C.-J. Yao, C.-M. Yang, S.-E. Chuang et al., "Targeting PMLRAR $\alpha$ and oncogenic signaling pathways by chinese herbal mixture Tien-Hsien liquid in acute promyelocytic leukemia NB4 cells," Evidence-Based Complementary and Alternative Medicine, vol. 2011, Article ID 984154, 11 pages, 2011.

[8] K. K. Chan, T. J. Yao, B. Jones et al., "The use of chinese herbal medicine to improve quality of life in women undergoing chemotherapy for ovarian cancer: a double-blind placebocontrolled randomized trial with immunological monitoring," Annals of Oncology, vol. 22, no. 10, pp. 2241-2249, 2011.

[9] A. Sun, J. S. Chia, C. P. Chiang et al., "The chinese herbal medicine Tien-Hsien liquid inhibits cell growth and induces apoptosis in a wide variety of human cancer cells," Journal of Alternative and Complementary Medicine, vol. 11, no. 2, pp. 245-256, 2005.

[10] H. Boon, M. Stewart, M. A. Kennard et al., "Use of complementary/alternative medicine by breast cancer survivors in Ontario: prevalence and perceptions," Journal of Clinical Oncology, vol. 18, no. 13, pp. 2515-2521, 2000.

[11] M. A. Richardson, T. Sanders, J. L. Palmer, A. Greisinger, and S. E. Singletary, "Complementary/alternative medicine use in a comprehensive cancer center and the implications for oncology," Journal of Clinical Oncology, vol. 18, no. 13, pp. 25052514, 2000.

[12] J. Shen, R. Andersen, P. S. Albert et al., "Use of complementary/alternative therapies by women with advanced-stage breast cancer," BMC Complementary and Alternative Medicine, vol. 2, no. 1, article no. 8, p. 8, 2002.

[13] B. Gerber, C. Scholz, T. Reimer, V. Briese, and W. Janni, "Complementary and alternative therapeutic approaches in patients with early breast cancer: a systematic review," Breast Cancer Research and Treatment, vol. 95, no. 3, pp. 199-209, 2006.

[14] A. Sun, J. S. Chia, W. B. Wang, and C. P. Chiang, "Immunomodulating effects of "Tien-Hsien liquid" on peripheral blood mononuclear cells and T-lymphocytes from patients with recurrent aphthous ulcerations," American Journal of Chinese Medicine, vol. 32, no. 2, pp. 221-234, 2004.

[15] A. Sun, J. S. Chia, W. B. Wang, and C. P. Chiang, "“Tien-hsien liquid' can modulate antigen-stimulated cytokine production by T-cells isolated from patients with recurrent aphthous ulcerations," American Journal of Chinese Medicine, vol. 33, no. 4, pp. 559-571, 2005.

[16] H. Svensson, T. Hatschek, H. Johansson, Z. Einbeigi, and Y. Brandberg, "Health-related quality oflife as prognostic factor for response, progression-free survival, and survival in women with metastatic breast cancer," Medical Oncology. In press.

[17] L. C. Chen, B. R. Wang, Y. C. Chou, and J. H. Tien, "Drug utilization pattern of chinese herbal medicines in a general hospital in Taiwan," Pharmacoepidemiology and Drug Safety, vol. 14, no. 9, pp. 651-657, 2005.
[18] E. Ernst, "Herbal medicines put into context," British Medical Journal, vol. 327, no. 7420, pp. 881-882, 2003.

[19] T. S. Mok, W. Yeo, P. J. Johnson et al., "A double-blind placebocontrolled randomized study of chinese herbal medicine as complementary therapy for reduction of chemotherapyinduced toxicity," Annals of Oncology, vol. 18, no. 4, pp. 768774, 2007.

[20] C. Q. Li, L. C. He, H. Y. Dong, and J. Q. Jin, "Screening for the anti-inflammatory activity of fractions and compounds from atractylodes macrocephala koidz," Journal of Ethnopharmacology, vol. 114, no. 2, pp. 212-217, 2007.

[21] [No authors listed], "Astragalus membranaceus. Monograph," Alternative Medicine Review-A Journal of Clinical Therapeutics, vol. 8, no. 1, pp. 72-77, 2003.

[22] Y.-H. Kim, S.-J. Choo, I.-J. Ryoo, J.-S. Ahn, and I.-D. Yoo, "Eudesmanolides from taraxacum mongolicum and their inhibitory effects on the production of nitric oxide," Archives of Pharmacal Research, vol. 34, no. 1, pp. 37-41, 2011.

[23] J.-L. Ríos, "Chemical constituents and pharmacological properties of poria cocos," Planta Medica, vol. 77, no. 7, pp. 681691, 2011.

[24] X. Ran, L. Ma, C. Peng, H. Zhang, and L.-P. Qin, "Ligusticum chuanxiong Hort: a review of chemistry and pharmacology," Pharmaceutical Biology, vol. 49, no. 11, pp. 1180-1189, 2011.

[25] D. Gao, Q. Li, Y. Li et al., "Antidiabetic and antioxidant effects of oleanolic acid from Ligustrum lucidum ait in alloxaninduced diabetic rats," Phytotherapy Research, vol. 23, no. 9, pp. 1257-1262, 2009.

[26] Y. X. Sun, "Immunological adjuvant effect of awater-soluble polysaccharide, CPP, from the roots of codonopsis pilosula on the immune responses to ovalbumin in mice," Chemistry and Biodiversity, vol. 6, no. 6, pp. 890-896, 2009.

[27] B.-S. Ko, J. S. Jang, S. M. Hong et al., "Changes in components, glycyrrhizin and glycyrrhetinic acid, in raw glycyrrhiza uralensis fisch, modify insulin sensitizing and insulinotropic actions," Bioscience, Biotechnology and Biochemistry, vol. 71, no. 6, pp. 1452-1461, 2007.

[28] J. Lin, L. Wei, W. Xu, Z. Hong, X. Liu, and J. Peng, "Effect of hedyotis diffusa willd extract on tumor angiogenesis," Molecular Medicine Reports, vol. 4, no. 6, pp. 1283-1288, 2011.

[29] C. K. Wong, K. N. Leung, K. P. Fung, and Y. M. Choy, “The immunostimulating activities of anti-tumor polysaccharides from pseudostellaria heterophylla," Immunopharmacology, vol. 28, no. 1, pp. 47-54, 1994.

[30] W. He, L. Y. Chan, G. Zeng, N. L. Daly, D. J. Craik, and N. Tan, "Isolation and characterization of cytotoxic cyclotides from viola philippica," Peptides, vol. 32, no. 8, pp. 1719-1723, 2011. 


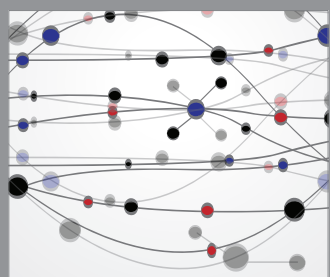

The Scientific World Journal
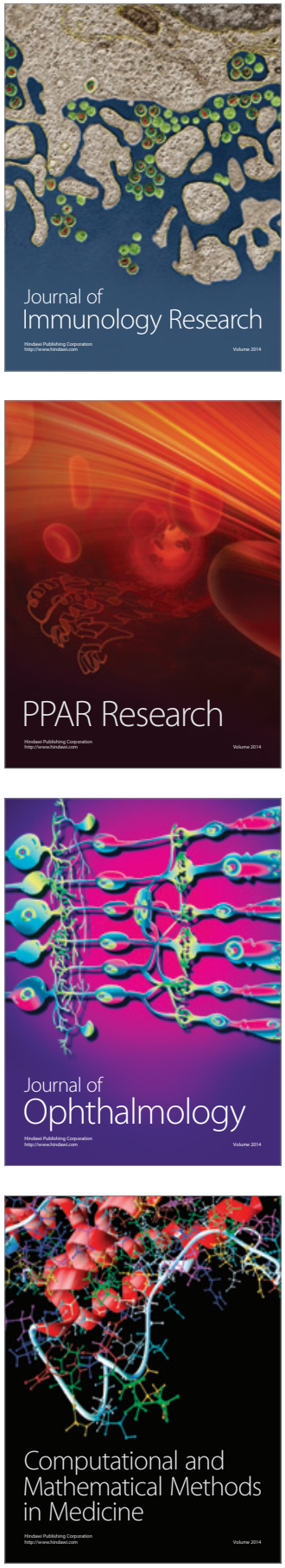

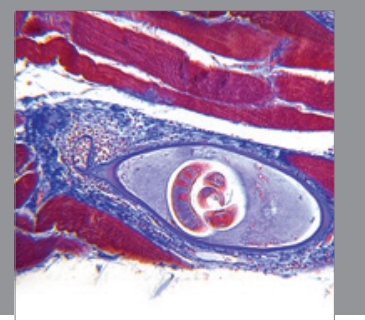

Gastroenterology

Research and Practice
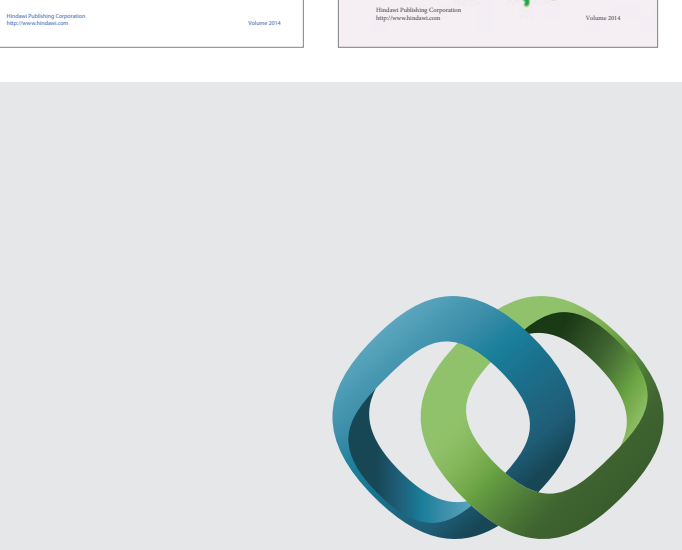

\section{Hindawi}

Submit your manuscripts at

http://www.hindawi.com
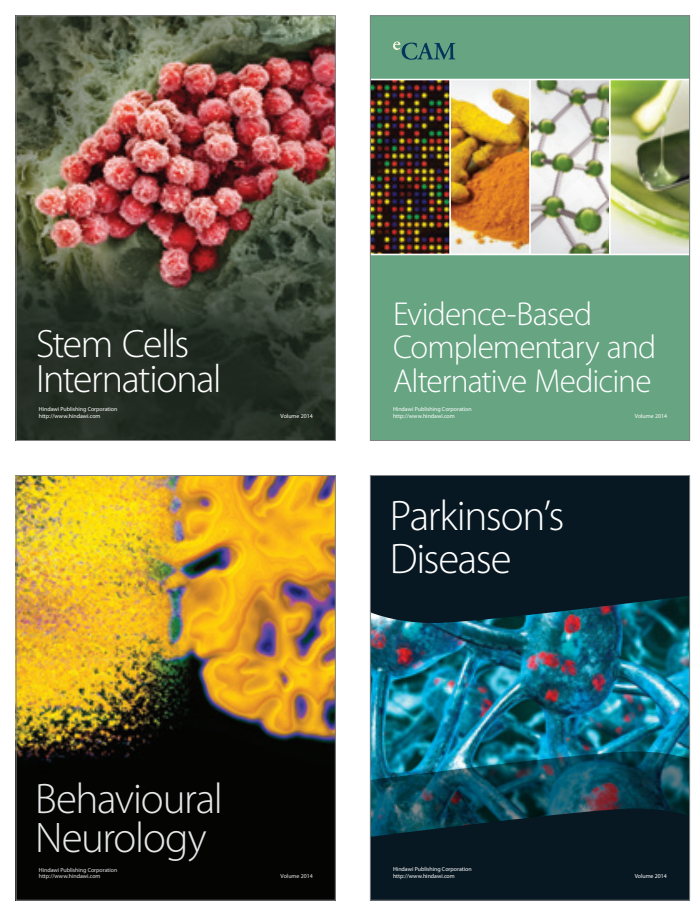

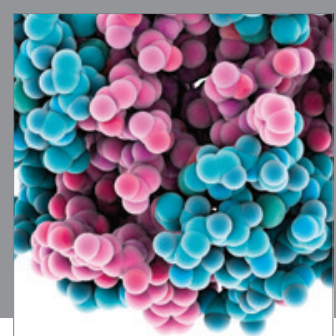

Journal of
Diabetes Research

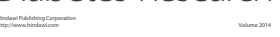

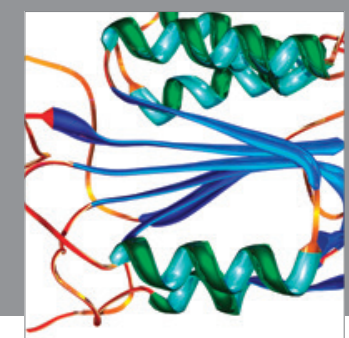

Disease Markers
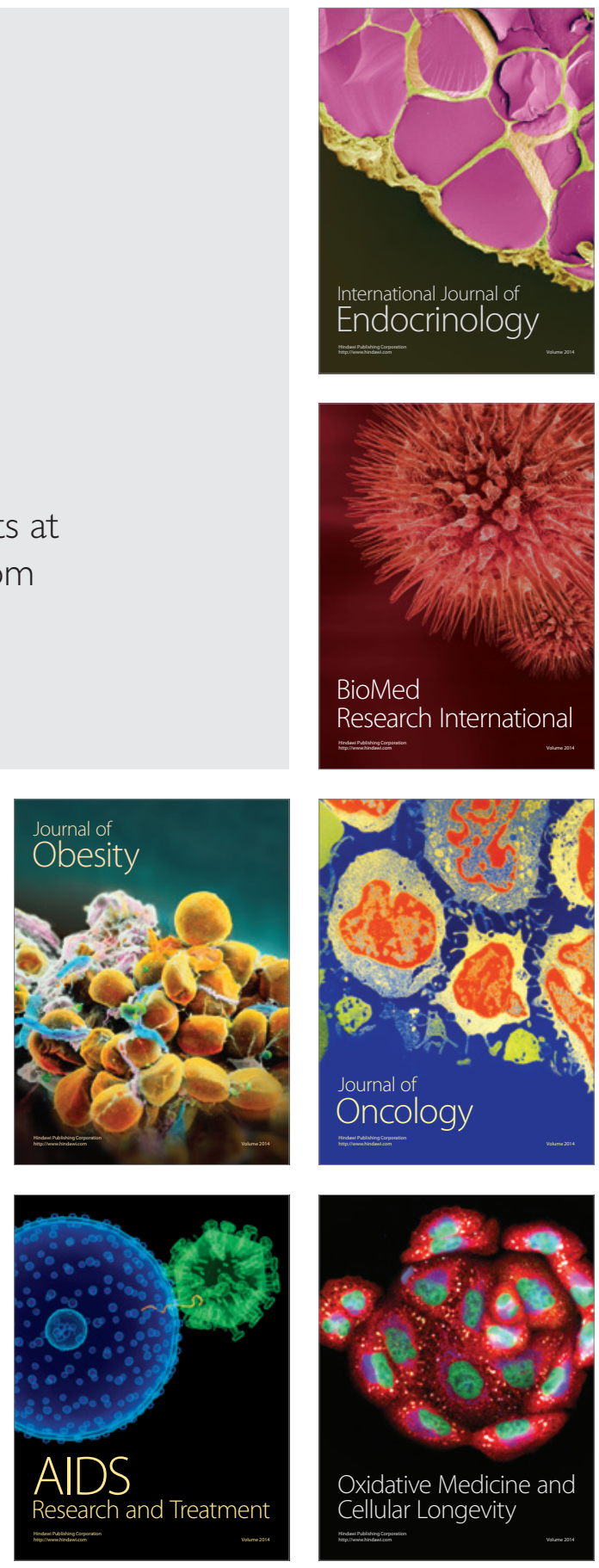